\title{
Application of Bio-Inspired Data Processing in Intelligent Transportation Systems
}

\author{
Amir Jabbari \\ Institute for Micro Sensors, \\ Actuators and Systems, \\ Department of Electrical \\ Engineering, University of Bremen \\ Bremen, Germany \\ ajabbari@imsas.uni-bremen.de
}

\author{
Hans-Jörg Kreowski \\ Research Group Theoretical \\ Computer Science \\ Department of Computer Science, \\ University of Bremen \\ Bremen, Germany \\ kreo@informatik.uni-bremen.de
}

\author{
Walter Lang \\ Institute for Micro Sensors, \\ Actuators and Systems, \\ Department of Electrical \\ Engineering, University of Bremen \\ Bremen, Germany \\ wlang@imsas.uni-bremen.de
}

\begin{abstract}
There has been a growing interest in using the soft computing techniques for distributed data processing in monitoring systems during last years; wireless sensor network is an example which can be utilized to record and process the environmental conditions in transportation systems. In this paper, by using knowledge based soft computing techniques, the reliability of the sensory records is evaluated autonomously in an intelligent transportation system; for this purpose, a new combinational architecture is applied including a bio-inspired local/global data approximation as well as a knowledge based data classification mechanism. The approximation mechanism is established on a least squares mechanism for global data prediction and a radial basis function network including immunological center selection to perform the local data approximations. To classify the records, a new probabilistic neural network including immune system features is developed. The proposed data processing technique is applicable for various intelligent data processing purposes.
\end{abstract}

Keywords-Soft computing; wireless sensor network; intelligent transportation system; bio-inspired data processing.

\section{INTRODUCTION}

An intelligent container is an intelligent transportation system to transport products under desired environmental conditions [1]; using radio frequency identification and wireless sensor networks, the instantaneous situation of the products are observed. The sensor network is comprised of many smart devices which are called sensor nodes to records temperature and humidity [2]. Application of soft computing techniques on wireless sensor networks leads to intelligent data processing in food transportation system.

Nowadays, there is a growing interest in application of bioinspired techniques which are derived from human biological system. Artificial neural networks (ANN) and artificial immune systems (IS) are two well-known algorithms to imitate the biological system to sense, process and make decisions either locally or globally; hybridization is another possibility to deal with the limitations of each bio-inspired algorithm for data processing. Neuro-immune system is a hybrid algorithm which is established on integrating immune system features to design and implement an efficient neural network [3]. In this paper, to process the sensory records, a two stage algorithm is developed including a data approximation and a classification algorithm according to Fig. 1. The least squares (LS) approach is a global approximation technique to predict the records in various ranges; however, to locally approximate the records, radial basis function (RBF) is an appropriate technique which leads to more accurate approximations within a specific range [4, 5]. The immune system can adjust the architecture of the radial basis function efficiently [3]. Combining a local and a global approximation algorithm can lead to a more sufficient prediction by observing the data range to switch between the local and global architectures at each instance.

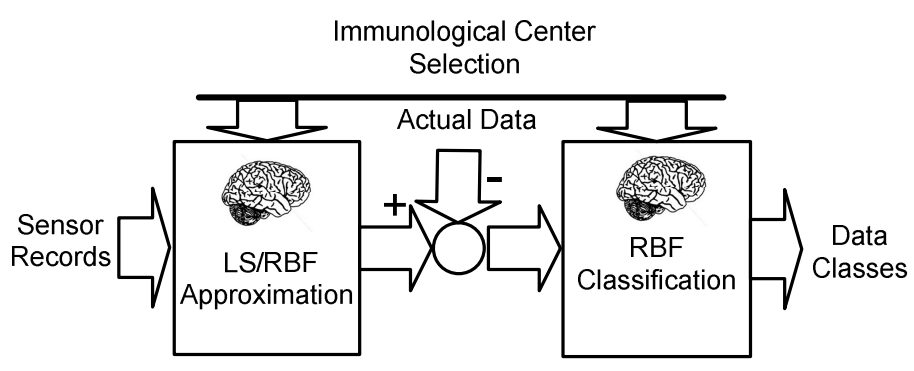

Fig. 1. A two stage data processing architecture.

In this paper, a least squares approximator is combined with a neuro-immune system to approximate the records. To classify the difference between the approximated and actual values - so called residual- a probabilistic radial basis function is served; immunological center selection adjusts the position and number of centers in the neural network. The proposed techniques are compared and advantages are highlighted.

\section{RELATED WORK}

Distributed data processing in a wireless sensor network denotes the data collection by various sensor nodes to make the decisions locally. A soft computing technique could be developed to predict and classify the sensory records to perform the autonomous data processing. Estimating the records of the nodes has a crucial role in data processing $[6,7$, 8]. Then, the predicted data is compared to the actual data to be classified either as plausible or implausible [5, 9]. The implausibility is due to fault occurrence either locally or globally in the wireless sensor network. Artificial immune 
systems are derived from biological immune systems to defend the body against the threats considering the interaction between antibodies and antigens [3]. Anomaly detection, data mining and fault detection are main applications of the artificial immune systems [3]. Immune system features are integrated in either classical or modern techniques to improve the performance; an application is to design an optimized neural network by updating the weights or selecting the network parameters. Dasgupta compared artificial neural networks with artificial immune systems [10]. De Castro et al. focused on self-organizing neural networks including immune system features. Also, an immunological Boolean competitive network was developed for data processing [11]. An unsupervised approach was introduced to adjust the radial basis function networks using a Euclidean shape-space to represent the molecules [12]. To adjust the initial weights of a feedforward neural network, immune system was considered to set the network weights [13].

\section{SOFT COMPUTING IN INTELLIGENT TRANSPORTATION SYSTEM}

There are two architectures to process the records in a wireless sensor network; some records are processed in a decentralized manner for local decision making at each cluster. The other architecture denotes the global data processing architecture when a central platform deals with the general decisions considering various clusters. In this paper, the records of each three sensor nodes are processed using a soft computing platform (SCP); so, each cluster includes four sensor nodes.

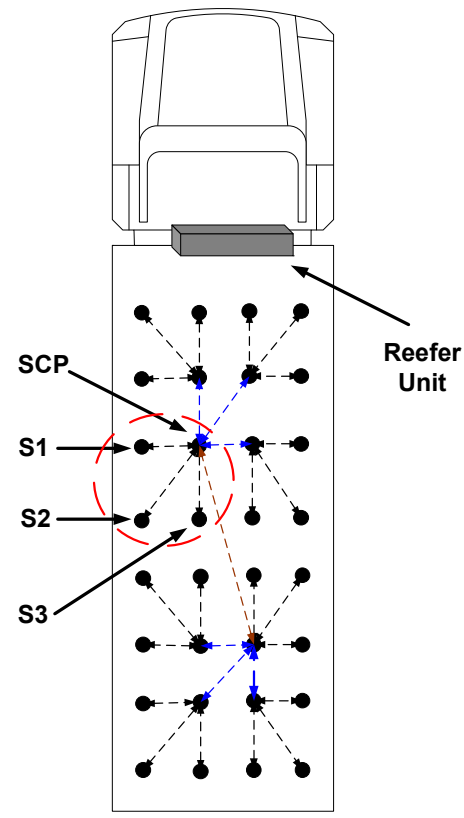

Fig. 2. Generalized data processing in a wireless sensor network.

The SCP collects the sensory records of every three sensor nodes at each instance and processes to evaluate the reliability of the records. Then, the records of each three SCP at three neighboring clusters are processed together using another SCP; in this way, all records are processed inside the transportation system. Fig. 2 shows the generalized data processing architecture inside a truck. A reefer unit warms up and cools down the truck by on-off cycles. The developed architecture was implemented inside a real truck which is shown in Fig. 3.

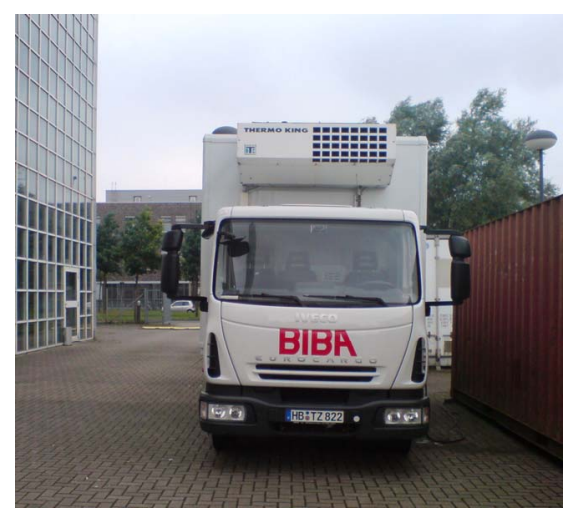

Fig. 3. A truck to transport the products.

The soft computing algorithm deals with data approximation and classification at each instance. One of the clusters according to Fig. 2 is chosen to implement and evaluate the data processing technique. Next section describes the details of the proposed data approximation architecture.

\section{A. Least Squares}

The least squares (LS) approximation is established on either determined or over-determined linear or nonlinear equations to model a system. In this paper, the LS approach denotes the four sequential records of the "under approximation sensor node" (for example when $S_{3}$ is considered as the under approximation sensor node) and the four sequential records of the neighboring sensor nodes $\left(S_{S C P}, S_{1}\right.$, and $\left.S_{2}\right)$ by an overdetermined matrix as shown in (1) [5];

$\left[\begin{array}{l}S_{3}(t) \\ S_{3}(t+1) \\ S_{3}(t+2) \\ S_{3}(t+3)\end{array}\right]=\left[\begin{array}{ccc}S_{S C P}(t) & S_{1}(t) & S_{2}(t) \\ S_{S C P}(t+1) & S_{1}(t+1) & S_{2}(t+1) \\ S_{S C P}(t+2) & S_{1}(t+2) & S_{2}(t+2) \\ S_{S C P}(t+3) & S_{1}(t+3) & S_{2}(t+3)\end{array}\right] \cdot K$

In (1), the four sequential values of parameters $S_{S C P}, S_{1}$, and $S_{2}$ are recorded and used to find the approximation coefficients ( $K=\left[\begin{array}{lll}k_{S C P} & k_{1} & k_{2}\end{array}\right]^{T}$ ) which are calculated by solving the over-determined set. With the new values of the approximation parameters $\left(S_{S C P}, S_{1}\right.$, and $\left.S_{2}\right)$ and the updated approximation coefficients ( $K$ vector), a new value of $S_{3}$ (at $\mathrm{t}+4)$ is calculated;

$$
S_{4}(t+4)=\left[S_{S C P}(t+4) \quad S_{1}(t+4) \quad S_{2}(t+4)\right] \cdot K
$$

This procedure continues and the coefficients are updated by the last four sequential equations. 


\section{B. Radial Basis Function with fixed centers}

Radial basis function networks are established on locally restricted functions (like Gaussian). The locally restricted functions are centered with the specific values representing a zone; then, the functions approximate any new value around the training range and according to the assigned centers.

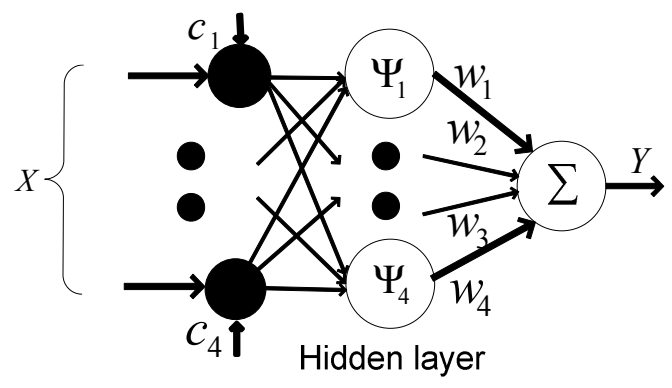

Fig. 4. Radial basis function architecture.

In RBF network (as shown in Fig. 4), an appropriate width and center should be assigned to each neuron in the hidden layer; also, the preliminary weights between the hidden layer and the output layer are calculated. After calculating the weighted input pattern, a nonlinear function is applied on the hidden layer. Thereafter, the weighted output of the hidden neurons is calculated $(Y)[4]$;

$$
\begin{aligned}
& Y=w_{i} \Psi_{i}\left(\left\|l_{i}-c_{i}\right\|\right) \\
& i=1,2,3, \ldots, m
\end{aligned}
$$

In (3), $l_{i}$ is the $\mathrm{i}$-th element of the input vector of the RBF network, $c_{i}$ is the i-th assigned center, $\|\cdot\|$ is the Euclidean norm, $\Psi_{i}$ is the i-th nonlinear function, $m$ is the number of neurons in the hidden layer, and $w_{i}$ is the i-th applied weight to the output of the i-th hidden neuron. To train the network, supervised and unsupervised approaches could be applied; equation (4) shows the procedure of updating the weights between hidden layer and output layer with "Gradient descent" algorithm, when fixed centers are selected (as shown in Fig. 4);

$$
w_{j k}(k+1)=w_{j k}(k)+\eta_{w} \cdot u_{i} \cdot\left(D_{j}-Y_{j}\right)
$$

In (4), $w_{j k}(k+1)$ is the new weight, $w_{j k}(k)$ is the current weight, $\eta_{w}$ refers to the associated learning factor for updating the weights, $u_{i}$ is the output of the i-th hidden neuron; $D_{j}$ and $Y_{j}$ are respectively the $\mathrm{j}$-th desired and the actual output of the network. Different network architectures were explored in order to find the optimum parameters; the results showed that when the records of a sensor node is approximated (as under approximation node) using three neighboring sensor nodes at each cluster, a hidden layer suffices including four hidden units to map the approximation parameters to the training target (actual value) during the training phase [5]. To approximate the new records, last four sequential records are selected to train the network.

\section{Radial Basis Function with immune system features}

According to the idea of biological immune systems, the artificial immune system is developed as an adaptive processing tool [3]. Antibodies (Ab) and Antigens (Ag) are two types of molecules; $\mathrm{Ab}$ molecules bind to $\mathrm{Ag}$ for their posterior elimination. For representing the artificial immune systems, the antibody and antigen vectors are shown as:

$$
\begin{aligned}
& A b=\left\langle A b_{1}, A b_{2}, \ldots, A b_{L}\right\rangle \\
& A g=\left\langle A g_{1}, A g_{2}, \ldots, A g_{L}\right\rangle
\end{aligned}
$$

Also, the affinity function which denotes the degree of match between the Ab-Ag vectors could be described by the following equation (Manhattan);

$$
D=\sum_{i=1}^{L}\left|A b_{i}-A g_{i}\right| \quad \text { (Manhattan distance) }
$$

In RBF network, finding the centers is a sensitive part of network design which have important role in accuracy of data approximation mechanism. An unsupervised training algorithm is applicable considering the immune system features; thus, (4) is replaced by a new criterion. The immunological center selection includes two main steps: at first, the number and location of the centers are selected randomly according to the data set; then, the dynamics of the centers population are controlled by a clonal selection algorithm; later, self-organizing individual centers are eliminated (suppression) and an unsupervised learning technique deals with the centers [12].

At first, $m$ centers are chosen including
$C=\left\{c_{1}, c_{2}, \ldots, c_{m}\right\} \quad$ instead of the input patterns
$X=\left\{x_{1}, x_{2}, \ldots, x_{N}\right\}$ (where $m$ is selected smaller than $\mathrm{N}$ ). The center elements are initialized randomly at the beginning and they are not expected to recognize each other. Two pairs are calculated at each time including $\mathrm{Ab}-\mathrm{Ag}$ affinities $d_{i j}$ $\left(d_{i j}=x_{i}-c_{j}\right)$ and interactions $s_{i j}\left(s_{i j}=c_{i}-c_{j}\right)$. Respectively, to control the death threshold, in purpose of eliminating extra centers as well as the self-recognizing elements from $\mathrm{C}$ (suppression), two thresholds including $\sigma_{d}$ (death threshold) and $\sigma_{s}$ (suppression threshold) are defined.

Each antibody corresponds to a favorite center; instead of that antibody each antigen is considered as relevant input set $\left(d_{i j}\right)$; also, antibodies (centers) should not detect and react against each other (that is why $s_{i j}$ is calculated). Firstly, for each input pattern, $d_{i j}$ is calculated; then, the highest affinity network centers are selected and reproduced (cloning) (6).

$$
\left\{\begin{array}{l}
d_{i j}=x_{i}-c_{j} \\
s_{i j}=c_{i}-c_{j}
\end{array}\right.
$$


Then, the centers with inferior affinity to the defined thresholds ( $\sigma_{d}$ and $\left.\sigma_{s}\right)$ are eliminated for clonal and network suppression, respectively. Finally, the affinity of the cells with any given input pattern $\left(x_{i}\right)$ can be improved by the following expression (biased mutation) [12]:

$$
c_{j}=c_{j}-\alpha_{j}\left(c_{j}-x_{i}\right)
$$

In (7), $\alpha_{j}$ is the unsupervised learning rate. When the affinity between centers and inputs are high, $\alpha_{j}$ is assumed to be small (around 0.1 in this paper); otherwise, it is great (0.9) when the affinity is small.

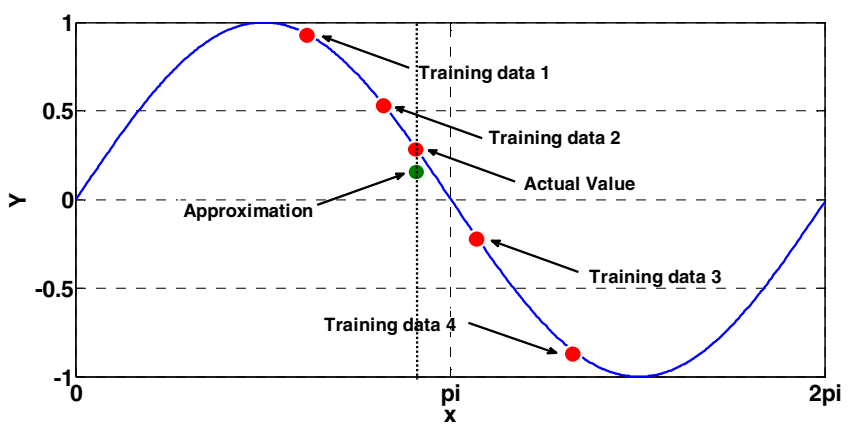

Fig. 5. Function approximation using RBF networks.

To evaluate the performance of the RBF network including either the fixed or immunologically selected centers, a sine wave is approximated. At each instance four (data) points are selected to train and approximate using both RBF networks including fixed and immunological center selection. 16 randomly generated patterns including four randomly generated points were used to establish the local approximation condition; an example is seen in Fig. 5. To compare the local approximation techniques, the probability $(P)$ of data approximation is calculated;

$$
P\left(x_{\text {approximation }}\right)=\frac{x_{\text {approximation }}}{x_{\text {actual }}} .100 \%
$$

The average value of $P\left(x_{\text {approximation }}\right)$ for 16 randomly generated patterns is $83.6 \%$ using RBF network with fixed centers while it goes up to $97.4 \%$ when the immunological center selection is applied according to Table I.

TABLE I. COMPARISON OF RBF APPROXIMATION TECHNIQUES

\begin{tabular}{|c|c|}
\hline Local Data Approximation Technique & Accuracy (\%) \\
\hline RBF with fixed centers & $83.6 \%$ \\
\hline RBF with immunological center selection & $97.4 \%$ \\
\hline
\end{tabular}

\section{Bio-inspired data classification}

Artificial neural network (ANN) classifiers have different advantages and disadvantages in comparison with the other classification approaches; they are not restricted to any especial function, but finding the appropriate function and training set has the key role in performance of the network; it is possible to apply the probability features which makes the probabilistic neural network an ideal mechanism for classification [5].

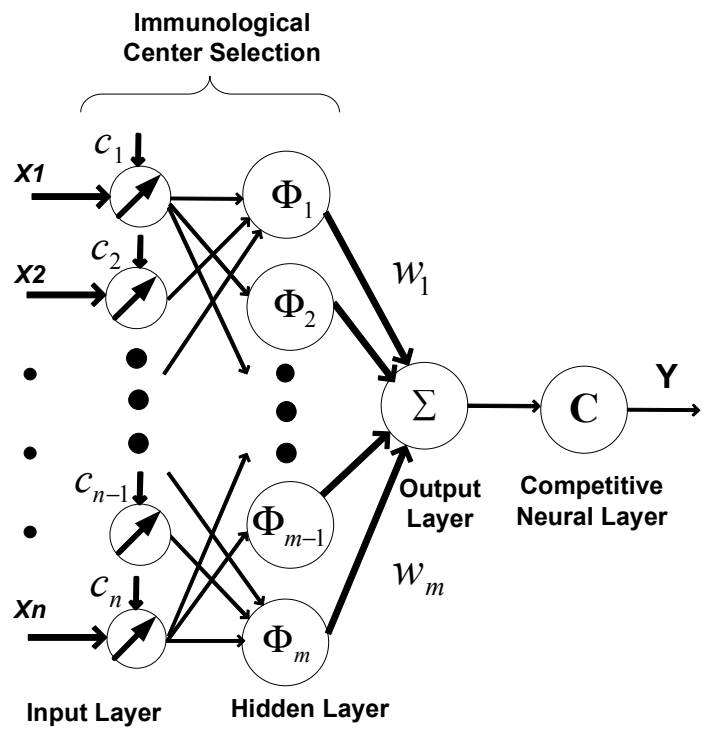

Fig. 6. Probabilistic RBF-immune network for data classification

For data classification, the centers are selected immunologically as described before. Fig. 6 illustrates the developed probabilistic neural network including a competitive layer (C) to compare and select the highest calculated values. In (9), $Y_{k}$ denotes the greatest output values from all hidden neurons and $\mathrm{m}$ denotes to the number of centers.

$$
\begin{aligned}
& \mathrm{Y}_{\mathrm{k}}(1) \geq \mathrm{Y}_{\mathrm{i}}(1) \\
& \mathrm{i}=1,2,3, \ldots . ., \mathrm{m} \\
& \text { IV. EXPERIMENTAL RESULTS }
\end{aligned}
$$

\section{EXPERIMENTAL RESULTS}

In this section, the proposed algorithms are implemented on the wireless sensor network to record and process the environmental conditions inside the truck (as seen in Fig. 3). The main objective of the experiments is to compare the proposed local/global data approximation technique (including least squares and RBF-immune system) with the least squares.

The experimental set up includes Imote2 sensor nodes [14], which are positioned inside the truck according to Fig. 2. All experiments were carried out at the cluster including $S_{S C P}, S_{1}$, $S_{2}$, and $S_{3}$; every 30 seconds, the SCP collects the records and processes the records of $S_{1}, S_{2}$, and $S_{3}$.

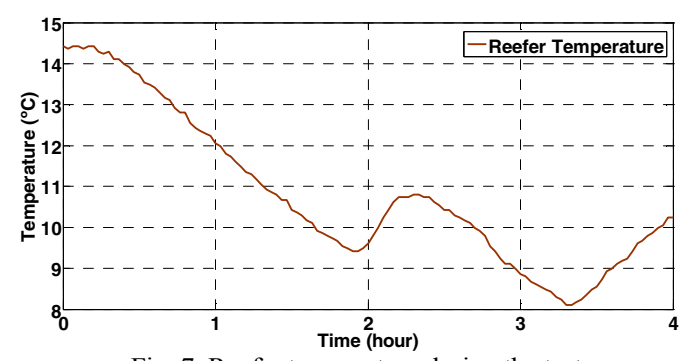

Fig. 7. Reefer temperature during the test. 
The test duration is about 4 hours and the reefer unit adjusts the environmental conditions according to some arbitrary set points between $8-16{ }^{\circ} \mathrm{C}$ to establish the real transportation conditions with different trends. The average ambient temperature is about $14.7{ }^{\circ} \mathrm{C}$. The reefer unit temperature is seen in Fig. 7 during the test.

\section{A. Data Approximation}

The temperature changes of the reefer unit vary the environmental conditions at different positions inside the truck. The difference between the actual and approximated value is calculated as the approximation residual. The average of the resultant approximation residuals for $S_{1}, S_{2}$, and $S_{3}$ are seen in Fig. 8 using the least squares approximation technique as well as the local/global approximation mechanism. According to the Fig. 8, the resultant data approximation value using local/global approximation mechanism leads to more accurate results in comparison to the least squares technique.

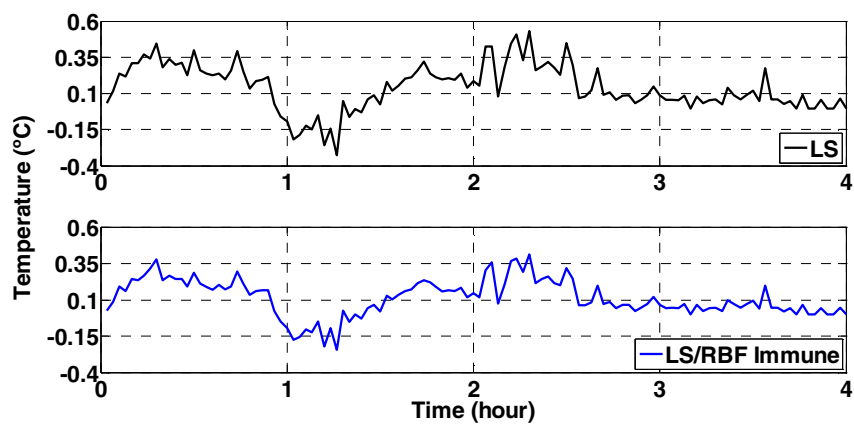

Fig. 8. Average of the approximation residuals using LS vs. LS/RBF-immune.

To compare the approximation values, "root mean squares" (RMS) of the average approximation residuals are calculated using (10);

$$
\operatorname{RMS}(\widetilde{Y})=\sqrt{\frac{\sum_{i=1}^{n}(Y-\widetilde{Y})^{2}}{n}}
$$

In (10), $Y$ and $\widetilde{Y}$ are the average of the actual and approximated values at the cluster, respectively; $n$ refers to the number of the approximated records.

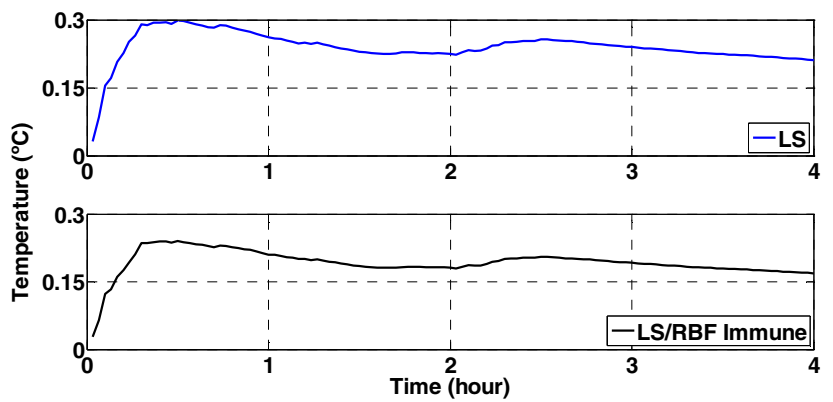

Fig. 9. RMS of the average approximation residual

Fig. 9 shows the computed RMS of data approximation using LS and LS/RBF-immune mechanism. The RMS of the average approximation residual is about $0.212{ }^{\circ} \mathrm{C}$ using LS approach; however, the RMS value of LS/RBF-immune reaches to $0.168^{\circ} \mathrm{C}$.

\section{B. Data Classification}

To classify the records, the bio-inspired data classification mechanism observes the RMS value of the average approximation residuals at SCP; exceeding the normal class needs to be investigated by the classification mechanism. Then, three Manhattan affinity functions are calculated; each affinity function denotes the Manhattan distance between the actual and approximated values at each node considering the last four sequential records. According to the calculated affinity functions $(A F)$, the following ranges are defined to classify the records (as illustrated in Fig. 10).

1. Class 1: Normal $\left(A F<0.75\left({ }^{\circ} \mathrm{C}\right)\right)$

2. Class 2: Unknown $\left(0.75<A F<1.25\left({ }^{\circ} \mathrm{C}\right)\right)$

3. Class 3: Abnormal $\left(1.25<A F\left({ }^{\circ} \mathrm{C}\right)\right)$

To deal with the uncertainty of the data approximation mechanism, $2^{\text {nd }}$ class is defined to investigate whether exceeding the normal condition is either due to the insufficient data approximation or fault occurrence (like battery discharge of a sensor node). When the approximated value of a sensor node deviates significantly from the actual value at a cluster, the computed affinity function will show huge deviation from normal condition (class 3); otherwise, when the calculated affinity function remains in the unknown area, a radial basis function network is used. To classify the $2^{\text {nd }}$ class for a sensor node, correlation factor is calculated between that sensor node and the neighboring nodes at the cluster.

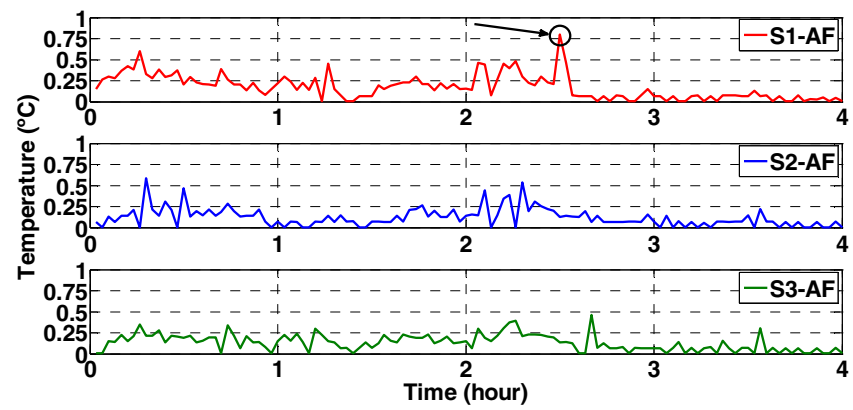

Fig. 10. Affinity functions between the actual records and approximations.

Fig. 10 shows an example, when the affinity function of $S_{1}$ once exceeds the normal class (about $0.783{ }^{\circ} \mathrm{C}$ ); however, the deviation of the approximated value from the actual records is not huge and needs to be evaluated by the classification mechanism.

The probabilistic RBF-immune network is trained using the data classes. Table II shows the provided data to train the classification network; at first, the absolute sliding correlation factor is caudated using (11).

$$
\operatorname{Corr}\left(S_{i}, S_{j}\right)=\left|\frac{\sum\left(s_{i}-\bar{s}_{i}\right) \cdot\left(s_{j}-\bar{s}_{j}\right)}{\sqrt{\left(s_{i}-\bar{s}_{i}\right)^{2} \cdot \sum\left(s_{j}-\bar{s}_{j}\right)^{2}}}\right|
$$


$\operatorname{Corr}\left(S_{i}, S_{j}\right)$ denotes the absolute sliding correlation factor between $S_{i}$ and $S_{j}$; also, $S_{i}$ and $s_{j}$ are the elements of those two sets respectively including the last four sequential records; $\bar{S}_{i}$ and $\bar{S}_{j}$ are their averages.

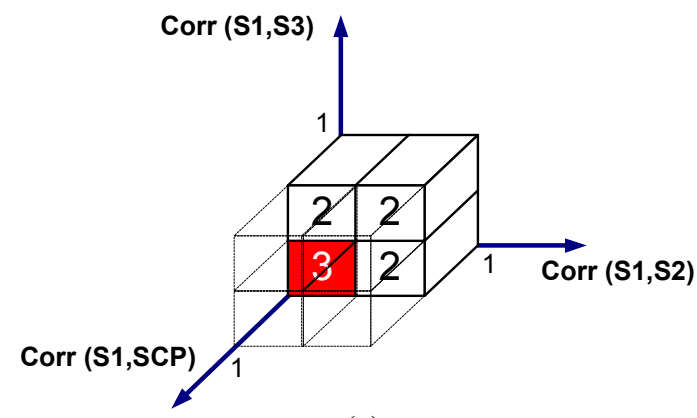

(a)

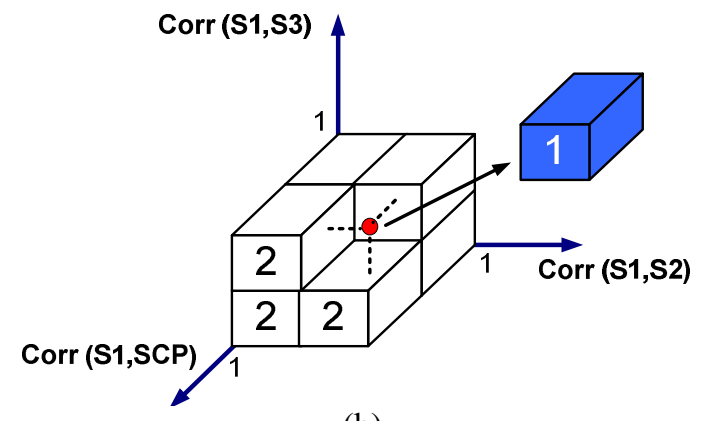

(b)

Fig. 11. Data classification range.

The resultant absolute sliding correlation factor of the $S_{1}$ within the unknown range is seen in Fig. 11; the absolute sliding correlation factor between $S_{1}$ and the neighboring sensor nodes including $S_{2}, S_{3}$ and $S C P$ are $0.84,0.91$ and 0.81 , respectively; therefore, despite exceeding the normal class, the approximated value is classified as class 1 .

TABLE II. TRAINING RBF-IMMUNE CLASSIFIER

\begin{tabular}{|c|c|}
\hline $\begin{array}{c}\text { Case } \\
\text { (Corr (S1,S2), Corr (S1,S3), Corr (S1,SCP)) }\end{array}$ & Class \\
\hline$(0.25,0.25,0.25)$ & 3 \\
\hline$(0.25,0.25,0.75)$ & 2 \\
\hline$(0.25,0.75,0.25)$ & 2 \\
\hline$(0.25,0.75,0.75)$ & 2 \\
\hline$(0.75,0.25,0.25)$ & 2 \\
\hline$(0.75,0.25,0.75)$ & 2 \\
\hline$(0.75,0.75,0.25)$ & 2 \\
\hline$(0.75,0.75,0.75)$ & 1 \\
\hline
\end{tabular}

\section{CONCLUSION}

In this paper, a novel bio-inspired data processing technique was implemented on a wireless sensor network to evaluate the reliability of the records. The proposed algorithm was developed to process the sensory records in an intelligent transportation system. The data approximation includes a local and a global data approximation technique. A radial basis function network was selected to design the local approximation mechanism including the immune system features to combine with a least squares approximation mechanism. Furthermore, a probabilistic RBF-immune network classified the approximation residual at each instance.

\section{ACKNOWLEDGMENT}

This research was supported by the German Research Foundation (DFG), as part of the Collaborative Research Centre 637 on "Autonomous Logistic Processes".

\section{REFERENCES}

[1] R. Jedermann, C. Behrens, D. Westphal, and W. Lang. "Applying autonomous sensor systems in logistics - Combining sensor networks, RFIDs and software agents," Sensors and Actuators A, vol. 132, 2006, pp. 370-375.

[2] N. Mahalik, Sensor Networks and Configuration: Fundamentals, Standards, Platforms, and Applications, Springer, USA, 2007, pp. 20.

[3] D. Dasgupta, Artificial Immune Systems and Their Applications, Ed., Springer-Verlag, 1999.

[4] M. M. Gupta, L. Jin, and N. Homma, Static and Dynamic Neural Networks: From Fundamentals to Advanced Theory, Wiley-IEEE: NJ, USA, 2003.

[5] A. Jabbari, R. Jedermann, R. Muthuraman, and W. Lang, "Application of Neurocomputing for Data Approximation and Classification in Wireless Sensor Networks," Sensors Journal, 2009, vol. 9, pp. 30563077.

[6] A. Swami, Q. Zhao, and Y. Hong, Wireless Sensor Networks: Signal Processing and Communications Perspectives, John Wiley and Sons, 2007, pp. 65.

[7] E.-O.Blass, J. Horneber, and M. Zitterbart, "Analyzing Data Prediction in Wireless Sensor Networks," IEEE Vehicular Technology Conference, Singapore, 2008, pp. $86-87$.

[8] A. Heshmati and M. Reza Soleymani, "An Energy-Efficient Cooperative Algorithm for Data Estimation in Wireless Sensor Networks," Canadian Conference on Electrical and Computer Engineering, Canada, 2007, pp. $928-931$.

[9] A. Jabbari, R. Jedermann, and W. Lang, "Application of Data Approximation and Classification in Measurement Systems," IEEE international conference on computational intelligence for measurement systems and applications, Istanbul, Turkey, 2008, pp. 64-69.

[10] D. Dasgupta, "Artificial Neural Networks and Artificial Immune Systems: Similarities and Differences," Proc. of the IEEE Systems, Man and Cybernetics, FL, USA, 1997, pp. 873-878.

[11] L. N. de Castro, F. J. Von Zuben, and G.A. de Deus, "The construction of a Boolean competitive neural network using ideas from immunology," Neurocomputing, vol. 50, 2003, pp. 51-85.

[12] L. N. De Castro and F. J. Von Zuben, "An Immunological Approach to Initialize Centers of Radial Basis Function Neural Networks," Proc. of Brazilian Congress on Neural Networks, Florianópolis, Brazil, 2001, pp. 79-84.

[13] L. N. De Castro and F. J. Von Zuben, "An Immunological Approach to Initialize Feedforward Neural Network Weights," Proc. of International Conference on Artificial Neural Networks and Genetic Algorithms, Prague, Czech Republic, 2001, pp. 126-129.

[14] Crossbow Homepage. Available online: http://www.xbow.com/, accessed date: 20.02.10. 